\title{
Erratum to: Perseveration in a spatial-discrimination serial reversal learning task is differentially affected by MAO-A and MAO-B inhibition and associated with reduced anxiety and peripheral serotonin levels
}

Peter Zhukovsky ${ }^{1,6}$ - Johan Alsiö ${ }^{1,2,6}$ • Bianca Jupp ${ }^{1,3,6}$ - Jing Xia ${ }^{1,6}$ - Chiara Giuliano ${ }^{1,6}$. Lucy Jenner ${ }^{1,6}$. Jessica Griffiths ${ }^{1,6}$ • Errin Riley ${ }^{1,6}$ - Sajeed Ali ${ }^{1,6}$ • Angela C. Roberts ${ }^{1,4}$. Trevor W. Robbins ${ }^{1,6}$ • Jeffrey W. Dalley ${ }^{1,5,6}$

Published online: 7 July 2017

(C) Springer-Verlag GmbH Germany 2017

Erratum to: Psychopharmacology 234 (9-10): 1557 - 1571 DOI 10.1007/s00213-017-4569-x

The name of Chiara Giuliano was incorrectly spelled in the original version of this article.

The online version of the original article can be found at http://dx.doi:10. 1007/s00213-017-4569-x

Jeffrey W. Dalley

jwd20@cam.ac.uk

1 Behavioural and Clinical Neuroscience Institute and Department of Psychology, University of Cambridge, Cambridge, UK

2 Department of Neuroscience, University of Uppsala, Uppsala, Sweden

3 Florey Institute of Neuroscience and Mental Health, University of Melbourne, Parkville, VIC, Australia

4 Department of Physiology, Development and Neuroscience, University of Cambridge, Cambridge, UK

5 Department of Psychiatry, University of Cambridge, Cambridge, UK

6 Department of Psychology, University of Cambridge, Downing Street, Cambridge CB2 3EB, UK 